\title{
Risk of Essure microinsert abdominal migration: case report and review of literature
}

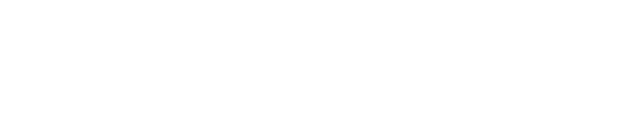

\author{
Giuseppe Ricci',2 \\ Stefano Restaino ${ }^{2}$ \\ Giovanni Di Lorenzo' \\ Francesco Fanfani' \\ Federica Scrimin' \\ Francesco P Mangino' \\ 'Institute for Maternal and Child \\ Health, IRCCS "Burlo Garofolo", \\ Trieste, Italy; ${ }^{2}$ Department of Medical \\ Sciences, University of Trieste, \\ Trieste, Italy
}

\begin{abstract}
Purpose: To report a case of Essure microinsert abdominal migration and literature review. Methods: A 41-year-old woman was counseled to undergo Essure sterilization. The procedure was hampered by the presence of endometrial cavity adhesions, obscuring left tubal ostium. By using microscissors the adhesions were progressively lysed. Since the procedure had become very painful, the patient required general anesthesia. Once adhesion lysis was completed, the tubal ostium was well visible. Both devices were then easily introduced into the fallopian tubes. At the end of the procedure, five coils were visible on the right side and five coils on the left side, as recommended.
\end{abstract}

Results: The 3-month hysterosalpingogram follow-up suspected abdominal migration of the left device. Laparoscopy confirmed the device displacement in the left lower abdominal quadrant. Both fallopian tubes and the uterus appeared normal. No signs of perforation were detected. The device was embedded into the omentum, but it was easily removed. Bilateral tubal sterilization was performed by bipolar coagulation.

Conclusion: There are only 13 cases, including the present, of Essure abdominal migration in the literature. In most cases, abdominal displacement of the microinsert is asymptomatic and does not induce tissue damage. However, in some cases, it may cause a severe adverse event, requiring major surgery. Therefore, removal of the migrated device should be performed as soon as possible. Moreover, during presterilization counseling, the patient should also be correctly informed about the risk of this rare but relevant complication, as well as about the surgical interventions that could be required to solve it.

Keywords: abdominal migration, Essure, hysteroscopic sterilization, hysteroscopy, tubal sterilization

\section{Introduction}

The Essure system was approved in November 2001 by the European Health Office and in November 2002 by the US Food and Drug Administration (FDA). According to the manufacturer, about 750,000 women have had it implanted to date. Many studies have shown that Essure is a safe and highly effective method of sterilization with a good patient compliance. ${ }^{1}$ However, with the increase in its use, it has become clear that this method is not without complications, which, in some cases, can also require a major surgical procedure. ${ }^{2}$ Abdominal migration of the device is a very rare complication of Essure sterilization. A recent analysis of adverse events reported in the Manufacturer and User Facility Device Experience (MAUDE) database (a system mandated by the FDA for postmarket surveillance) includes 90 cases of uterine perforation and 33 cases of microinsert malposition. ${ }^{3}$ However, no percentage of abdominal device migration is provided. Therefore, the actual risk of Essure abdominal migration is not known. There are very few cases reported in the literature and only some of these
Correspondence: Giuseppe Ricci Institute for Maternal and Child Health, IRCCS “Burlo Garofolo", Via dell'Istria 65/I, 34137 Trieste, Italy

Tel +390403785 589

Fax+3904076I 266

Email ricci@burlo.trieste.it 
are fully described. There was no uniform management of this complication, especially in asymptomatic patients. The aim of this article is to report a case of abdominal Essure migration and a review of the literature. The management of abdominal Essure migration is also discussed.

\section{Case report}

A 41-year-old woman, gravida 3, para 2, was counseled to undergo Essure sterilization. She had dyslipidemia and her body mass index was 25 . Four years earlier she had suffered a stroke while using oral contraceptives. This event caused persistent mild neurological deficits. Cycles were regular and gynecological examination was normal. The hysteroscopic sterilization was hampered by the presence of endometrial cavity adhesions obscuring left tubal ostium. By using microscissors the adhesions were progressively lysed. Since the procedure had become very painful, the patient required general anesthesia. Once adhesion lysis was completed, the tubal ostium was well visible. Both devices were then easily introduced into the fallopian tubes. At the end of the procedure, five coils were visible on the right side and five coils on the left side, as recommended. The procedure was completed in 25 minutes. The same day the patient was discharged and she was doing well.

During the following weeks, the patient did not complain of any symptoms. The 3-month hysterosalpingogram (HSG) follow-up showed that the right tube was occluded and the device was correctly placed (Figure 1). Conversely, the left

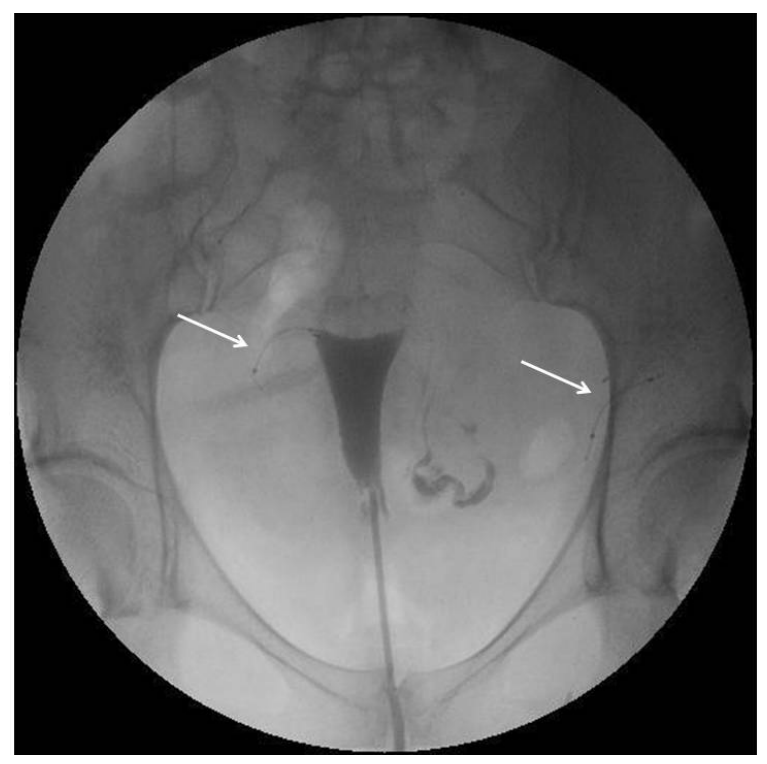

Figure I Hysterosalpingogram.

Notes: Hysterosalpingogram showing tubal occlusion on the right side with correct location of the device (right arrow) and a patent tube on the left side. The left device is abnormally positioned (left arrow). tube was patent and the device was abnormally located in the left side of the pelvis, away from the tubal distal extremity. Although the patient was asymptomatic, she was counseled to undergo laparoscopy in order to remove the device and complete tubal sterilization. Laparoscopy showed that the left device was displaced in the left lower quadrant, embedded into the omentum, without signs of inflammation or adhesions. The uterus and the fallopian tubes appeared normal, and no signs of perforation were detected. The device was easily removed by blunt and sharp dissection. Bilateral tubal sterilization was subsequently performed by bipolar coagulation. The patient's postoperative course was uneventful.

\section{Discussion}

Essure abdominal displacement is a very rare complication of hysteroscopic sterilization. The real frequency of this event is difficult to estimate because not all cases are reported. We reviewed the literature by searching in the Ovid/MEDLINE, PubMed, Scopus, and Google Scholar databases for all the articles published from January 2002 to December 2013. The following terms were used: "Essure microinsert", "hysteroscopic sterilization", "transcervical sterilization", "tubal occlusion", "perforation", "abdominal migration", and "complication". We also reviewed all the references cited in the papers. The cases of tubal or uterus perforation, or "perforation" without other specification, in which abdominal displacement of the device was not explicitly mentioned, were excluded. All types of studies, including case reports, case series, controlled clinical trials, and randomized controlled trials, were considered. There were no language restrictions. Animal studies were excluded. The literature search identified 59 articles, 51 of which were excluded, leaving eight articles describing 12 cases of Essure abdominal migration for final analysis (Figure 2). ${ }^{4-11}$

To the best of our knowledge, the case that we have observed is the 13 th reported case in literature. It shows some peculiarities in respect to the other cases reported. Although the procedure was complicated and a tubal perforation might have been supposed, after the procedure and in the following period, the patient remained asymptomatic and the laparoscopy bore no evidence of uterine or tubal injury. Moreover, even if the microinsert was completely embedded into the omentum, no signs of inflammation or adhesions were observed.

Table 1 summarizes all reported cases of Essure abdominal migration and allows the drawing of some suggestions to manage this rare complication. 


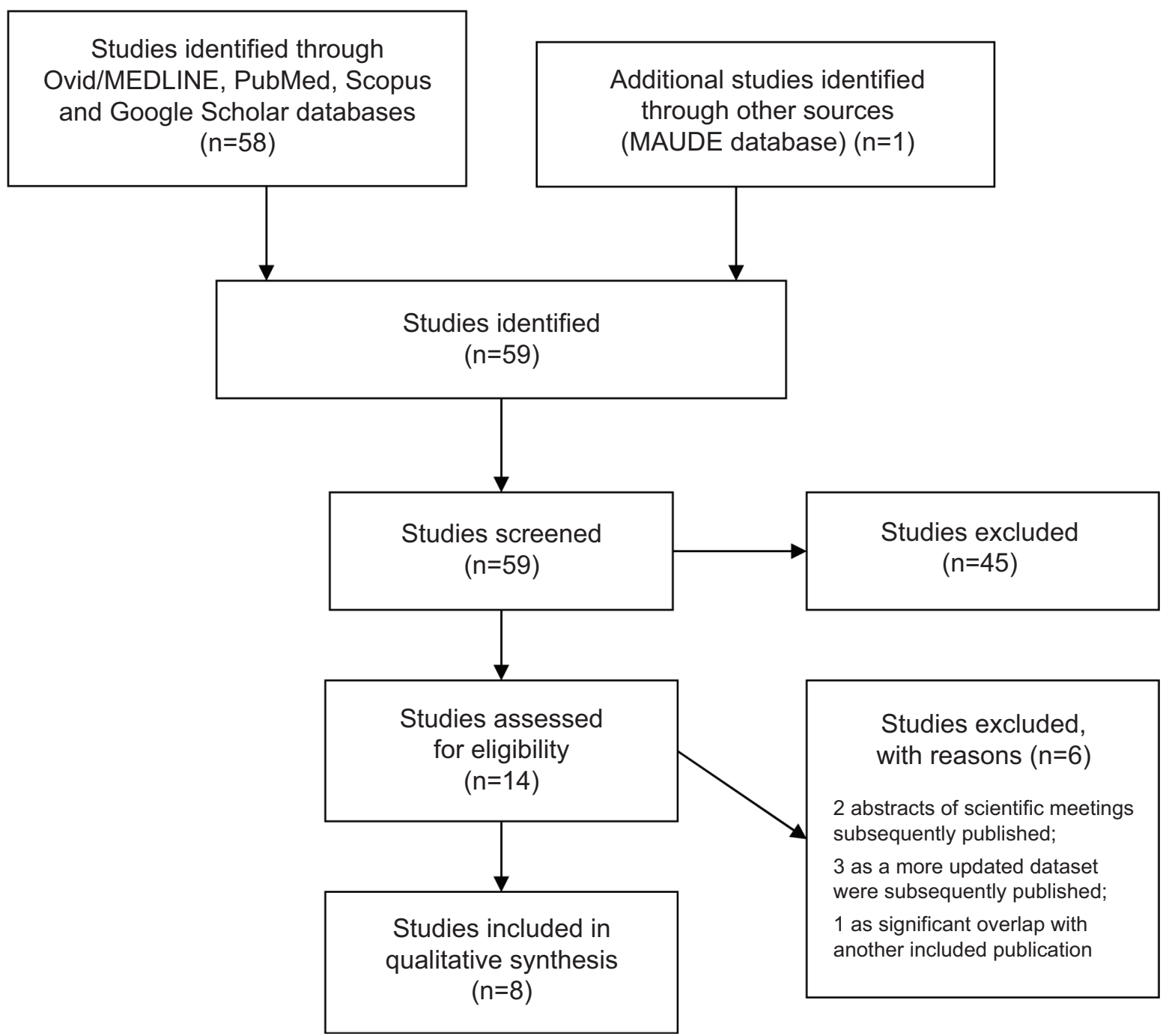

Figure 2 Flow diagram for study selection.

Abbreviation: MAUDE, Manufacturer and User Facility Device Experience.

Various factors may influence the risk of hysteroscopic sterilization complication among which the operator's experience and the anatomical anomalies represent the most relevant ones. ${ }^{1,2}$ The operator's experience significantly influences the rate of Essure migration. In fact, in the initial series, the reported rate is approximately $1 \%-2 \%,{ }^{5}$ whereas, in large series, including long-term experience, the rate is very low. ${ }^{9}$ Among more than 4,300 cases observed in 7 years, Povedano et $\mathrm{al}^{9}$ report a rate of only $0.04 \%$. Anatomical impediments such as ostium stenosis, occlusion, no visible ostium/scarring, and extremely lateral/tortuous tubes, as well as uterine pathology (polyps, adhesions), may make the placement of Essure difficult. ${ }^{1,2}$ In the present review, in three out of eight patients, the device displacement might be explained by the presence of laterally sited ostia, ${ }^{5}$ by tubal resistance, ${ }^{7}$ or by endometrial cavity adhesions (present case). On the contrary, in five out of eight cases, abdominal migration occurred after an uneventful procedure. ${ }^{5,6,8,10,11}$

A further risk factor of complication may be represented by general anesthesia, as it may, in fact, hide the pain due to tubal perforation. Unfortunately, except for our case, in the other reviewed cases, the analgesic or anesthetic protocol used was not specified. However, the abdominal migration was asymptomatic in most of the reported cases. ${ }^{5,6,8,10,11}$ Only our patient required general anesthesia due to unbearable pain during the procedure, whereas in the first case described by Vellayan et $\mathrm{al}^{5}$ the pain that occurred during the procedure settled spontaneously. In the $80 \%$ of cases reviewed in this paper, the displacement of the device was diagnosed at 3-month follow-up HSG (Table 1). In these cases, the postoperative course was uncomplicated, except for in the first patient observed by Vellayan et $\mathrm{al}^{5}$ who 6 days after 


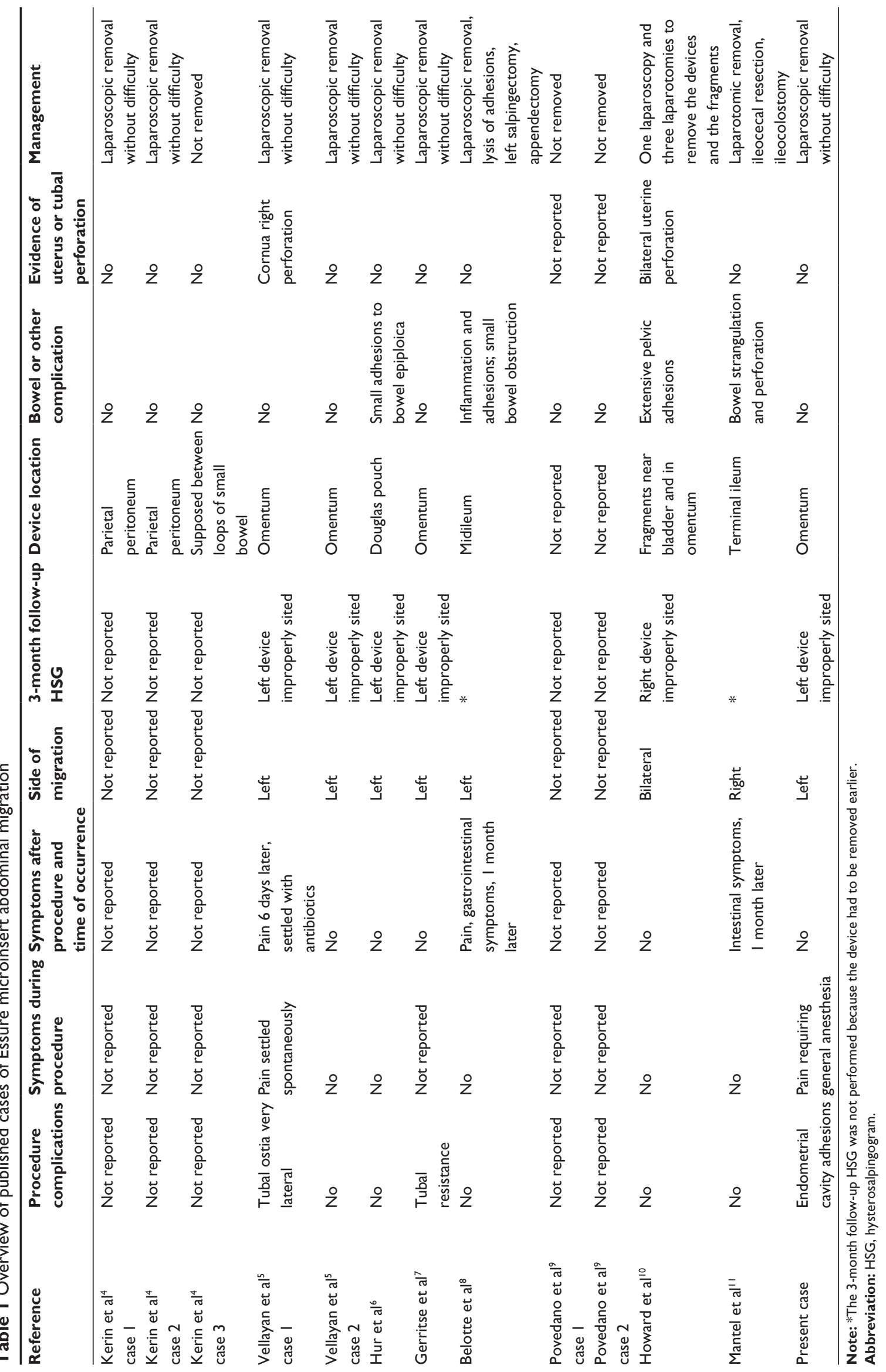


the procedure complained of pain, which was settled after antibiotic administration. In two cases the diagnosis was made 1 month after an uncomplicated procedure because the patients complained of rapidly worsening abdominal symptomatology. ${ }^{8,11}$ In one of these cases, a small bowel obstruction was diagnosed by laparoscopy. ${ }^{8}$ Lysis of adhesions, removal of the Essure microinsert, appendectomy, and left salpingectomy were performed, leading to the resolution of the symptoms. ${ }^{8}$ In the second case, due to nausea, vomiting, abdominal pain, and X-ray findings, the patient underwent a laparotomy. ${ }^{11}$ A microinsert had transversed across the mesentery and ensnared a loop of the terminal ileum, causing strangulation and perforation of the bowel wall. ${ }^{11}$ Thus, the migrated device was removed and an ileocecal resection was performed with a side-to-side ileocolostomy. ${ }^{11}$

As shown in Table 1, in asymptomatic patients almost all authors decided to remove the displaced device. The removal was easily performed in all cases, except for one case in which the device was fragmented and extensive pelvic adhesions had formed. ${ }^{10}$ In this case, four subsequent surgeries were needed to remove, in order, the right microinsert that was found to be perforating through the uterine fundus, the left microinsert extending from the endometrial cavity through the left myometrium into the peritoneal fat, and, ultimately, the microfragments embedded into tissue adjacent to the bladder and into the omentum. Only Kerin et $\mathrm{al}^{4}$ reported three cases where Essure was left in situ after having noticed that the pelvic organs were healthy and normal. The authors did not report later complications for these patients. ${ }^{4}$

In some cases, the abdominal migration of the device is certainly due to uterus or tubal perforation, because the perforation is visible at surgical intervention. In the present review, only in two cases out of nine did the surgeon detect a uterine perforation. ${ }^{5,10}$ In the remaining cases, although the surgeon performed an accurate evaluation of uterus and tubes, no signs of perforation or scars were identified (Table 1). In such cases, the possibility that migration occurred through the fallopian tube cannot be excluded. An alternative hypothesis is that the surgeon may have perforated the tube and the insert continued into the abdomen, and the tube just healed in the interval before the surgery. However, if the procedure is uncomplicated and the recommended number of coils are left in the endometrial cavity, the probability of this event should be very low.

Different locations of the device were reported (Table 1). In four cases it was adherent to, or entrapped in, the omentum; in two cases it was on peritoneum parietal; in two cases it was on the ileum; and in one case it was found in the Douglas pouch. Fragments of one device were found embedded in the tissue near the bladder and in the omentum. ${ }^{10}$ Considering that the Essure microinsert should stimulate only benign tissue growth, ${ }^{2}$ it is not clear why in some cases it instead induces inflammation and adhesions, leading to major complications. ${ }^{8,10,11}$ In some cases, hypersensitivity reactions to nickel or to other elements of the microinsert might be involved in the pathogenesis of this complication, as already suggested for orthopedic implant failure. ${ }^{12}$ Finally, in six cases out of eight, the migrated device was the one inserted in the left tube (Table 1). Although the sample size is too small to draw any conclusions, it can be hypothesized that this might be due to the more difficult approach to left tubal ostia by right-handed operators.

\section{Conclusion}

This review suggests that in the case of a complicated procedure, the right placement of both devices should be ascertained by adequate imaging and the patient should be strictly followed up. Moreover, in the case of occurrence of pelvic or abdominal symptomatology, bowel obstruction or perforation should be immediately excluded. In the case of asymptomatic device displacement detected at 3-month follow-up, a laparoscopy should be performed and the device should be removed. Bilateral laparoscopic sterilization should also be performed. The removed device should be carefully inspected and compared with a new, unused one through a magnifying glass (because of the small size coils) in order to exclude that fragments were left in the abdomen. In cases where the original placement was complicated, the opportunity of removing the contralateral device should also be considered. Alternatively, a follow-up of the patient should be made necessary. Finally, we advise that during presterilization counseling the patient should also be correctly informed about the risk of this rare but relevant complication, as well as about the surgical interventions that could be required to solve it. The rate and the severity of complications of other available sterilization methods should also be taken into account and made comparable with Essure sterilization for an informed choice.

\section{Author contributions}

All authors provided substantial contributions to conception and design, acquisition of data, or analysis and interpretation of data, and contributed to drafting the article and revising it critically for important intellectual content. All authors provided final approval of the version to be published, and agreement to be accountable for all aspects of the work in 
ensuring that questions related to the accuracy or integrity of any part of the work are appropriately investigated and resolved.

\section{Disclosure}

The authors report no conflicts of interest in this work.

\section{References}

1. Lessard CR, Hopkins MR. Efficacy, safety, and patient acceptability of the Essure ${ }^{\mathrm{TM}}$ procedure. Patient Prefer Adherence. 2011;5:207-212.

2. Hodges KR, Swaim LS. Hysteroscopic sterilization in the office setting. Obstet Gynecol Clin North Am. 2013;40(4):671-685.

3. Al-Safi ZA, Shavell VI, Hobson DT, Berman JM, Diamond MP. Analysis of adverse events with Essure hysteroscopic sterilization reported to the Manufacturer and User Facility Device Experience database. J Minim Invasive Gynecol. 2013;20(6):825-829.

4. Kerin JF, Cooper JM, Price T, et al. Hysteroscopic sterilization using a micro-insert device: results of a multicentre phase II study. Hum Reprod. 2003;18(6): 1223-1230

5. Vellayan M, Baxter A, Connor M, Brown V. The Essure ${ }^{\mathrm{TM}}$ hysteroscopic sterilisation procedure: initial experience in Sheffield, UK. Gynecol Surg. 2006;3:303-307.
6. Hur H, Mansuria SM, Chen BA, Lee TT. Laparoscopic management of hysteroscopic Essure sterilization complications: report of 3 cases. J Minim Invasive Gynecol. 2008;15(3):362-365.

7. Gerritse MB, Veersema S, Timmermans A, Brölmann HA. Incorrect position of Essure microinserts 3 months after successful bilateral placement. Fertil Steril. 2009;91(3):930.e1-e5.

8. Belotte J, Shavell VI, Awonuga AO, Diamond MP, Berman JM, Yancy AF. Small bowel obstruction subsequent to Essure microinsert sterilization: a case report. Fertil Steril. 2011;96(1):e4-e6.

9. Povedano B, Arjona JE, Velasco E, Monserrat JA, Lorente J, CasteloBranco C. Complications of hysteroscopic Essure ${ }^{\mathbb{E}}$ sterilisation: report on 4,306 procedures performed in a single centre. BJOG. 2012;119(7): 795-799.

10. Howard DL, Christenson PJ, Strickland JL. Use of intraoperative fluoroscopy during laparotomy to identify fragments of retained Essure microinserts: case report. J Minim Invasive Gynecol. 2012;19(5): 667-670.

11. Mantel HT, Wijma J, Stael AP. Small bowel obstruction and perforation after Essure sterilization: a case report. Contraception. 2013;87(1): 121-123.

12. Thomas P, Braathen LR, Dörig M, et al. Increased metal allergy in patients with failed metal-on-metal hip arthroplasty and peri-implant T-lymphocytic inflammation. Allergy. 2009;64(8):1157-1165.
Therapeutics and Clinical Risk Management

\section{Publish your work in this journal}

Therapeutics and Clinical Risk Management is an international, peerreviewed journal of clinical therapeutics and risk management, focusing on concise rapid reporting of clinical studies in all therapeutic areas, outcomes, safety, and programs for the effective, safe, and sustained use of medicines. This journal is indexed on PubMed Central, CAS,

\section{Dovepress}

EMBase, Scopus and the Elsevier Bibliographic databases. The manuscript management system is completely online and includes a very quick and fair peer-review system, which is all easy to use. Visit $\mathrm{http}: / /$ www.dovepress.com/testimonials.php to read real quotes from published authors. 